\title{
Mechanics Mechanism of Re-fracturing Propagation and Reorientation in Low Permeability Reservoirs
}

\author{
X.M. CHEN ${ }^{1,2} \&$ S.F. XUE ${ }^{1}$ \\ ${ }^{1}$ College of Pipeline and Civil Engineering, China University of Petroleum, Qingdao, Shandong, 266580, \\ China \\ ${ }^{2}$ Binnan Oil Production Plant, Shengli Oilfield Co., Ltd., China Pet rochemical Corporation, Binzhou \\ 256606, China
}

\begin{abstract}
Hydraulic fracturing is an important EOR technology of low permeability reservoirs which occupy a considerable proportion in China's oil reserves. If hydraulic fracturing fails, re-fracturing will be used in most oil-water wells. However, there are often a large number of inclusions in low-permeability reservoirs. Due to the complexity of the re-fracturing mechanism in low permeability reservoirs, the theoretical researches are far behind the field practices. Theoretical and experimental studies on the mechanical mechanism of re-fracturing are significant for the development of inclusion reservoirs.

Ground stress distribution is one of the major factors which affect fracture propagation after re-fracturing. In this paper line inclusion is seen as a thin bar, and the basic solution of elastic mechanics for line inclusion in infinite plane is used to solve the stress field. Stress field around artificial fracture which is influenced by single line inclusion is deduced. The interference problem of single line inclusion and artificial fracture under internal pressure is reduced to a set of Cauchy singular integral equations. In addition, a validation test with a large size $(500 \mathrm{~mm} \times 500 \mathrm{~mm} \times 500 \mathrm{~mm})$ true tri-axial test equipment is carried out. The results show that, the inclusions reduce rock intensity, and induce reservoir to generate more complex fractures.
\end{abstract}

KEYWORD: Mechanical mechanism of re-fracturing; Low permeability reservoir; Evolution of stress; Fracturing physical simulation

\section{INTRODUCTION}

The re-fracturing technology, as an enhanced recovery technique, was first developed in the 1950s, and it has become one of the important means to improve the recovery of oil and gas fields. The initiation and propagation of new fracture are greatly influenced by the reservoir structural characteristics during re-fracturing. Present studies mainly focus on the interference problems between artificial fracture and natural ones in natural fractured reservoirs. Many experiments have shown that fracture may present different state by the influence of various factors after the interference of artificial and natural fractures in natural reservoirs [1-6]. Zhou and Xue analyzed the impact of pre-existing fracture conductivity, stress state and injection rate on fracture path complexity [7]. On the basis of laboratory and field evidence, it is conceivable that several events may occur during the time period of the hydraulic fracture propagating toward the natural fracture [8]. However, there are numerous kinds of complex isolated inclusions in the reservoirs. The stress singularity may exist at the tip of the flake inclusions, and under certain conditions, it may also lead to the generation of micro-cracks [9]. Jin Yan et al. [10] analyzed the effects of salutatory barrier on hydraulic fracture propagation, but they have no further theoretical analysis.

At present, the inclusion theory is mainly used for composite materials. In this paper, inclusion theory was introduced for the inclusion reservoir. Stress intensity factor is derived at the tip of artificial fractures and inclusions. The direction of fracture propagation after re-fracturing can be judged by the maximum tensile stress criterion in defect reservoirs. In addition, the effects of isolated defects on the fracture shape after re-fracturing are verified by large size true tri-axial experiments.

\section{BASIC MODEL}

The basic model of stress distribution before refracturing in inclusion reservoir is shown as figure 1. The length of the inclusion is assumed far greater than its width, and the inclusion is seen as a line inclusion. For an elastic inclusion body, to better reflect the discontinuity of normal stress and tangential stress between the inclusion and rock 
matrix, the line inclusion is treaded as a thin bar. The Eshelby equivalent inclusion theory $[11,12]$ is used to solve the problem, only considering rock skeleton stress.

The stress field in the inclusion reservoir is composed of the following three parts:

(1) The stress field induced by the artificial fracture;

(2) The stress field induced by the line inclusion;

(3) Far-field stress.

The total stress state of elastic plane can be expressed as

$$
\begin{aligned}
& \sigma_{i j}(x, y)=\sigma_{\mathrm{a}, i j}(x, y)+\sigma_{\mathrm{d}, i j}(x, y)+\sigma_{\mathrm{w}, i j}(x, y) \\
& (i, j)=(x, y)
\end{aligned}
$$

where, $\sigma_{i j}(x, y)$ is the total stress field, MPa; $\sigma_{\mathrm{a}, i j}(x, y)$ is the stress field induced by artificial fracture, $\mathrm{MPa} ; \sigma_{\mathrm{d}, i j}(x, y)$ is the stress field induced by the line inclusion, MPa; $\sigma_{\mathrm{w}, i j}(x, y)$ is the farfield stress, MPa.

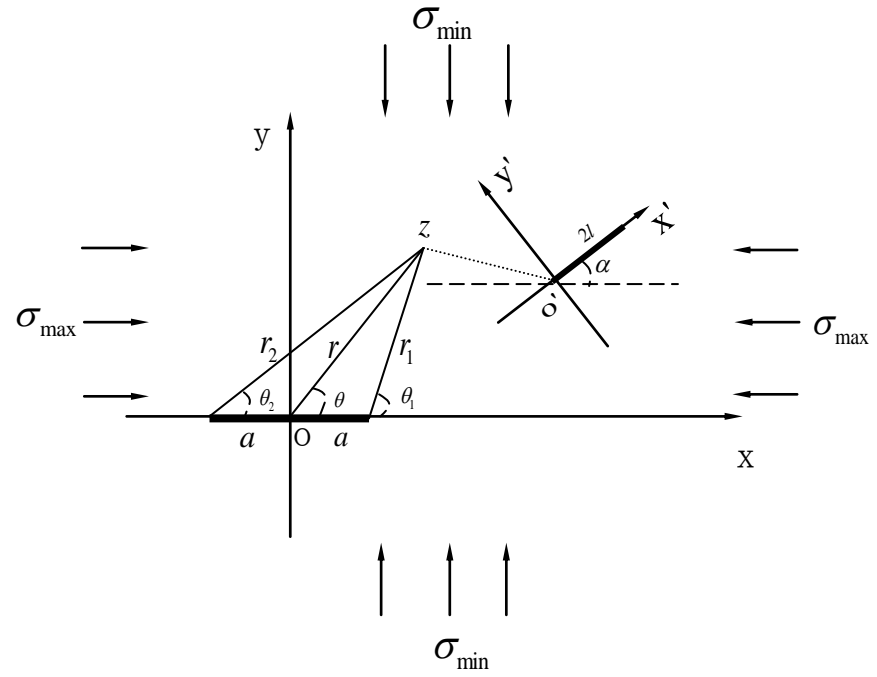

Figure 1. The stress distribution before re-fracturing in inclusion reservoir1

The boundary conditions are as follows,

(1) Total stress at the edge of the line inclusions needs to meet the displacement compatibility relations. That can be written in the local coordinate $x^{\prime} o^{\prime} y^{\prime}$ as follows,

$$
\begin{aligned}
& \frac{\partial u_{\mathrm{a}}{ }^{\prime}\left(x^{\prime},+0\right)}{\partial x^{\prime}}+\frac{\partial u_{\mathrm{d}}{ }^{\prime}\left(x^{\prime},+0\right)}{\partial x^{\prime}}+\frac{\partial u_{\mathrm{w}}{ }^{\prime}\left(x^{\prime},+0\right)}{\partial x^{\prime}}=\frac{\partial u_{\mathrm{d}} *\left(x^{\prime}\right)}{\partial x^{\prime}} \\
& \left(0<=x^{\prime}<=2 l\right) \\
& \frac{\partial v_{\mathrm{a}}{ }^{\prime}\left(x^{\prime},+0\right)}{\partial x^{\prime}}+\frac{\partial v_{\mathrm{d}}{ }^{\prime}\left(x^{\prime},+0\right)}{\partial x^{\prime}}+\frac{\partial v_{\mathrm{w}}{ }^{\prime}\left(x^{\prime},+0\right)}{\partial x^{\prime}}=\frac{\partial v_{\mathrm{d}} *\left(x^{\prime}\right)}{\partial x^{\prime}} \\
& \left(0<=x^{\prime}<=2 l\right)
\end{aligned}
$$

where, $u_{i}{ }^{\prime}$ and $v_{i}{ }^{\prime}$ are displacement components.

(2) The inner stress $p$ is exerted at the edge of artificial fracture. The direction is perpendicular to the fracture surface.

$$
\begin{aligned}
& \sigma_{\mathrm{a}, y y}(x, 0)+\sigma_{\mathrm{d}, y y}(x, 0)+\sigma_{\mathrm{w}, y y}(x, 0)=p \\
& (-a=<x<=a) \\
& \sigma_{\mathrm{a}, x y}(x, 0)+\sigma_{\mathrm{d}, x y}(x, 0)+\sigma_{\mathrm{w}, x y}(x, 0)=0 \\
& (-a=<x<=a)
\end{aligned}
$$

Based on inclusion theory, combined with the equations (2) and (3), we can obtain four Cauchy singular integral equations. After solving, the stress field at any point of the inclusion reservoirs can be expressed after a series of coordinate conversion. At the same time, we can deduce the stress intensity factor at the tip of artificial fracture and inclusions.

When the artificial fracture and line inclusions are disjoint, the stress intensity factor at the tip of the artificial fracture can be obtained by the following equations.

$$
\begin{aligned}
& K_{\mathrm{I}}(-a)=\frac{2 \mu}{\kappa+1} \lim _{x \rightarrow-a} \sqrt{2(a+x)} g(x) \\
& K_{\mathrm{I}}(a)=-\frac{2 \mu}{1+\kappa} \lim _{x \rightarrow a} \sqrt{2(a-x)} g(x)
\end{aligned}
$$

where, $a$ is the half-length of artificial fracture, $\mathrm{m} ; K_{\mathrm{I}}$ is the stress intensity factor of model I crack, $\mathrm{MPa} \cdot \sqrt{\mathrm{m}} ; \kappa$ is the elastic constant of rock matrix, and for plane strain problem, $\kappa=3-4 v$, where, $v$ is Poisson ratio; $g(x)$ is the dislocation density function of artificial fracture at $x=-a \sim+a, y=0$,

$$
\begin{aligned}
& g(x)=\frac{\partial}{\partial x}\left[u_{y}(x,+0)-u_{y}(x,-0)\right] \\
& (-a<x<a)
\end{aligned}
$$

In the local coordinate system, the stress intensity factor at the tip of line inclusions can be expressed as follows.

$$
\begin{aligned}
& K_{\mathrm{I}}{ }^{\prime}(0)=-\frac{\kappa-1}{2(\kappa+1)} \lim _{x^{\prime} \rightarrow 0} \sqrt{2 x^{\prime}} q\left(x^{\prime}\right) \\
& K_{\mathrm{I}}{ }^{\prime}(2 l)=\frac{\kappa-1}{2(\kappa+1)} \lim _{x^{\prime} \rightarrow 2 l} \sqrt{2\left(2 l-x^{\prime}\right)} q\left(x^{\prime}\right)
\end{aligned}
$$

where, $2 l$ is the length of the inclusion, $\mathrm{m} ; q\left(x^{\prime}\right)$ is the tangential constraint physical stress of the inclusion on rock matrix,

$$
q\left(x^{\prime}\right)=\sigma_{x y}\left(x^{\prime},-0\right)-\sigma_{x y}\left(x^{\prime},+0\right)
$$

Therefore, when we compare the above results with the critical stress intensity factor $K_{\text {IC }}$, we can judge the extension of the fractures in the inclusion reservoir. From equation (6) and (7), we can conclude that the stress intensity factor of the inclusion tip is affected by the local stress field around the inclusion. In the whole model, the local disturbance of the inclusion is controlled by the far field stress and the inner boundary pressure. Therefore, when the load changes, $K_{\mathrm{I}}{ }^{\prime}$ may be 
greater than $K_{\mathrm{IC}}$. And the new fracture may produce along the defect. The experiment will be done to prove it.

\section{EXPERIMENTAL VERIFICATION}

\subsection{Experimental equipments}

The experiments were performed in an improved large true tri-axial rock mechanics experiment system. Cubic model blocks of $500 \mathrm{~mm}$ on a side were positioned in the high-pressure cylinder for simulating in-situ stress conditions. The random defects were added in the samples, and the initial artificial fracture was simulated by a thin plastic sheet. The appearance of the high-pressure cylinder, which is one of the main equipments, is shown in figure 2 .

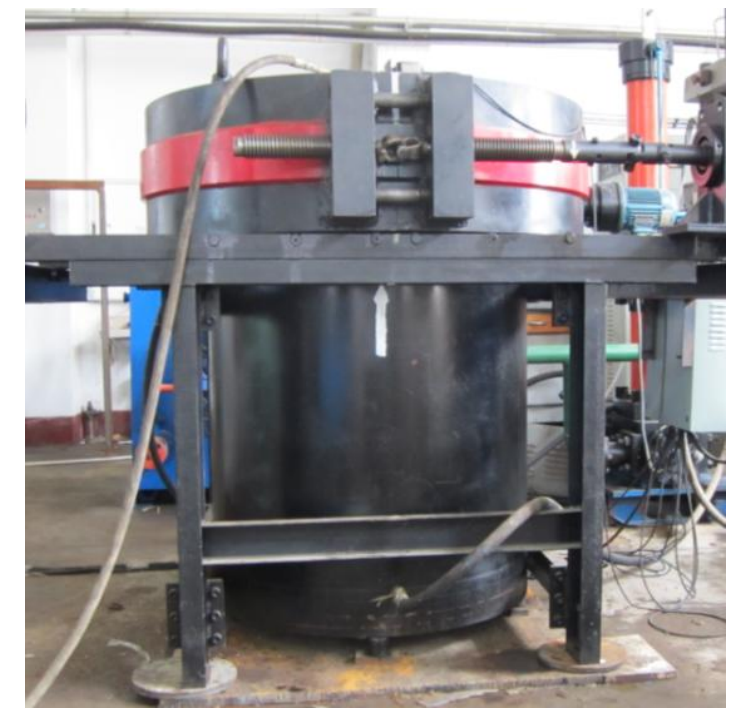

Figure 2 The appearance of the high-pressure cylinder

\subsection{The experiment process and results}

In fact, the critical stress intensity factor of the line inclusion is greatly difficult to measure. However, we can make a perceptual knowledge for the fracture propagation mechanism after re-fracturing in defect reservoirs by changing the size of the ground stress directly.

Table 1 Experimental stress data

\begin{tabular}{|c|c|c|c|c|c|c|}
\hline \multirow{2}{*}{$\begin{array}{c}\text { Sample } \\
\text { number }\end{array}$} & \multicolumn{3}{|c|}{ The first fracturing } & \multicolumn{3}{c|}{ The second fracturing } \\
\cline { 2 - 7 } & $\sigma_{\mathrm{x}}$ & $\sigma_{\mathrm{y}}$ & $\sigma_{\mathrm{z}}$ & $\sigma_{\mathrm{x}}$ & $\sigma_{\mathrm{y}}$ & $\sigma_{\mathrm{z}}$ \\
\hline $2^{\#}$ & 3 & 3 & 3 & 3 & 4 & 5 \\
\hline $4^{\#}$ & 4 & 4 & 4 & 1 & 2 & 3 \\
\hline $6^{\#}$ & 3 & 4 & 5 & & & \\
\hline
\end{tabular}

There are six samples designed for the experiments. The stresses of three samples is applied as shown in table 1 , where $\sigma_{x}$ and initial artificial fracture are in the same direction. Before re-fracturing experiment, we measured the rock mechanics parameters of the sample through a group of small samples. Elastic modulus of sample matrix is $2.4 \mathrm{GPa}$, Poisson ratio is 0.23 .

For example, when the confining pressure was $3 \mathrm{MPa}$ for $2^{\#}$ sample, a horizontal main fracture formed after the first fracturing. The highest pump pressure was $6.5 \mathrm{MPa}$. The second fracturing was carried out after changing the size of stress. A vertical main crack was created along the maximum horizontal principal stress direction. The highest pump pressure was $2.4 \mathrm{MPa}$ affected by the defects.

The strength of the rock is dispersed for defects of various scales in the rock, and the ability of resisting external force is weakened [13]. Under external loads, the defects of the sample will be activated. The activation defects extend under the load, and finally lead to mutual merger and samples are destructed. Therefore, when the loads are up to a certain threshold, random defects are activated in the defect reservoirs during the experiments. And more complex fractures may produce.
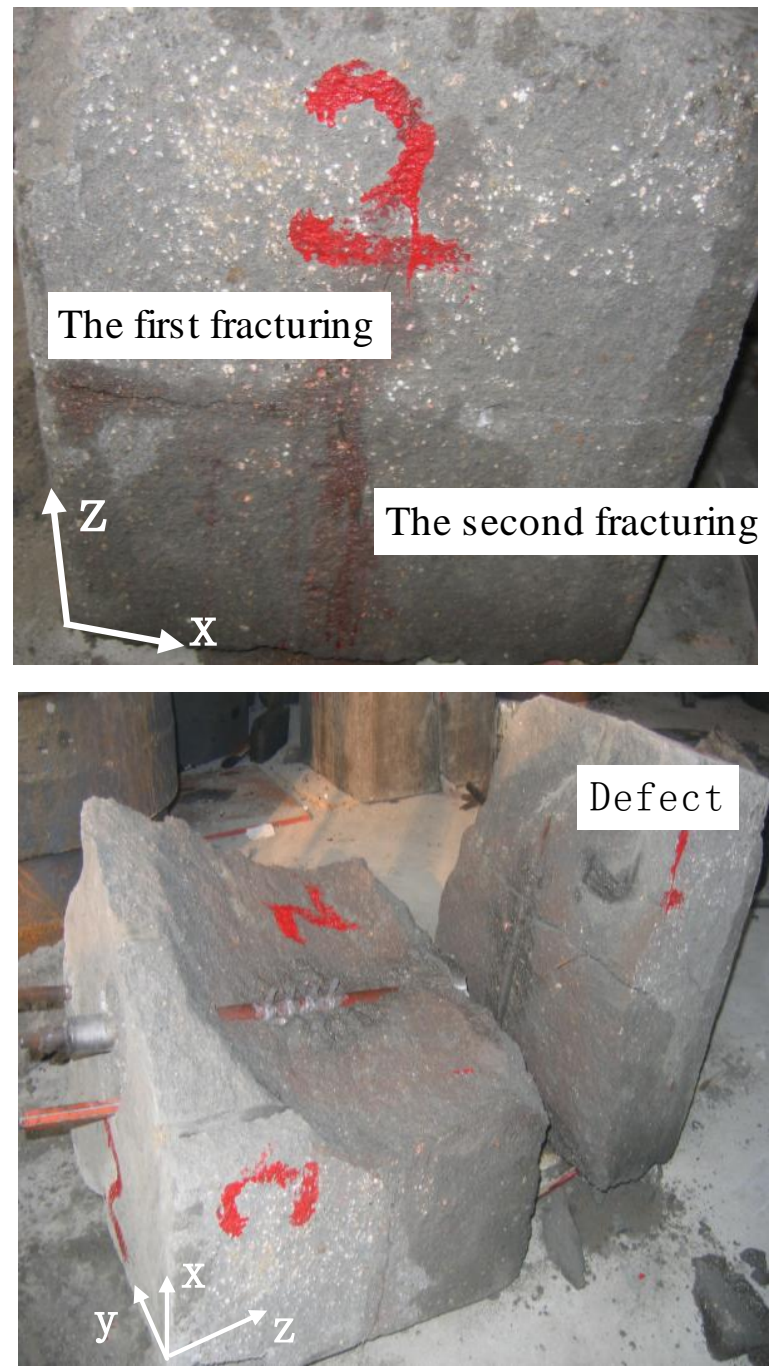

Figure 3 Experimental image of $2 \#$ sample after hydraulic fracturing 


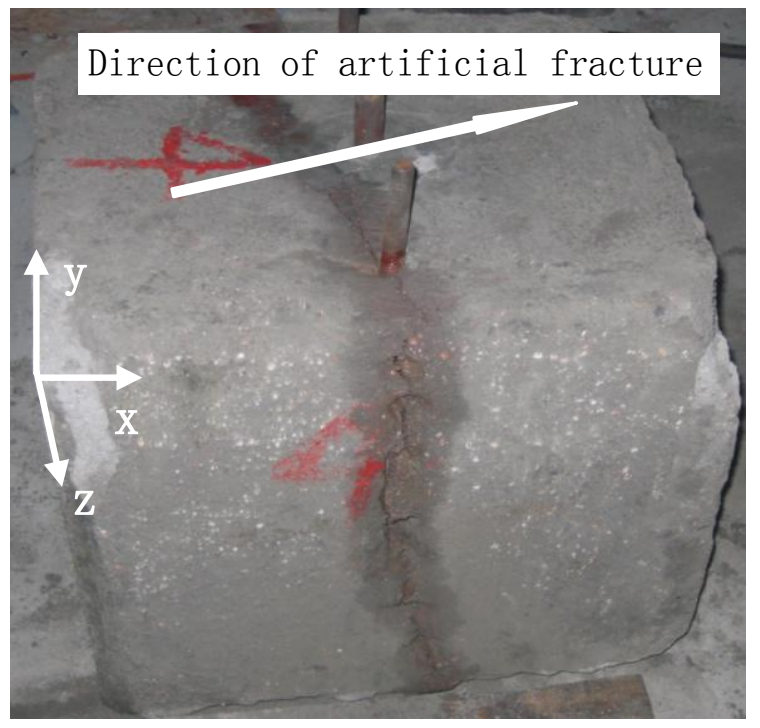

Figure 4 Experimental image of $4 \#$ sample after hydraulic fracturing

For 4\# sample, under the higher uniform confining pressure, the artificial fracture closed. And a new fracture produced along the direction perpendicular to the preset fracture which affected by the defects. The highest pump pressure was $6.2 \mathrm{MPa}$. When the size and orientation of the stresses were changed, the direction of the new fracture would not change, which still followed the direction of the maximum horizontal principal stress.

From the above experiments, we can conclude that the defect is one of the most important factors for the fracture morphology after re-fracturing in heterogeneous reservoir. However, whether the direction of the fracture propagation will be changed by the defect is finally directed by the stress distribution state in the reservoir, which meets the maximum tensile stress criterion. At the same time, we found that, under the higher uniform pressure stress, the direction of the new fracture may have no relation with the preset fracture in the defect reservoirs.

\section{CONCLUSION}

Theoretical research and indoor experiments have shown that the defect is one of the most important factors affecting the fracture distribution state after re-fracturing in the reservoir. From this paper, we can conclude as follows.

(1) Theory research shows that, in a certain stress condition, a new fracture may produce along the tip of the inclusion.
(2) The strength of the rock is dispersed for defects of various scales in the rock.

(3) Whether the presence of defects will cause the new fracture, to a great extent, is controlled by the far field stress.

(4) From the experiments, under some higher confining pressure, the defect may be the dominant factor of fracture propagation in the reservoirs.

\section{REFERENCES}

[1] Beugelsdijk L J L, Pater C J, Sato K. Experimental hydraulic fracture propagation in a multi-fractured medium. SPE 59419, 2000.

[2] Peacock D C P, Mann A. Controls on fracturing in carbonate rocks. SPE 92980, 2005.

[3] Warpinski N R, Lorenz J C, Branagan P T, Myal F R and Gall B L. Examination of a cored hydraulic fracture in a deep gas well. SPE 22876, 1993.

[4] Olson J E, Bahorich B, Holder J. Examining hydraulic fracture-natural fracture interaction in hydrostone block experiments. SPE 152618, 2012.

[5] Meng Chunfang. Hydraulic fracture propagation in prefractured natural rocks. SPE 140429, 2011.

[6] ZHOU Jian, CHEN Mian, JIN Yan, ZHANG Guang-qing. Experimental study on propagation mechanism of hydraulic fracture in naturally fractured reservoir. Acta Petrolei Sinica, 2007, 28(5): 109-113. (in Chinese)

[7] Zhou and Xue, Experimental investigation of fracture interaction between natural fractures and hydraulic fracture in naturally fractured reservoirs. SPE 142890, 2011.

[8] H.Gu, X.Weng, J.Lund, et al. Hydraulic fracture crossing natural fracture at nonorthogonal angles: a criterion and its validation. SPE 139984, 2012

[9] ZHANG Hong-tu, ZHE Xiao-li. Theory of inclusion and applications in the study of fracture. Acta Physica Sinica, 1981, 30(6): 761-773. (in Chinese)

[10] JIN Yan, CHEN Mian, ZHOU Jian, GENG Yu-di. Experimental study on the effects of salutatory barrier on hydraulic fracture propagation of cement blocks. Acta Petrolei Sinica, 2008, 29(2): 300-303. (in Chinese)

[11] HUANG Chun-ping, LI Zhong-hua. An approximate solution for the interaction forces between model I crack and an inclusion of arbitrary shape. Journal of Shanghai Jiaotong University, 2005, 39(1): 142-146. (in Chinese)

[12] ZHANG Ming-huan, TANG Ren-ji. Interaction between crack and elastic inclusion. Applied Mathematics and Mechanics, 1995, 16(4): 289-300. (in Chinese)

[13] DENG Yue-hong. Dimension stone strength and fractal characteristics of inner fault distribution. MINING R \& D. 2001, 21(6): 13-15. 